\title{
ON HOLOMORPHIC FUNCTIONS WITH CERTAIN EXTREMAL PROPERTIES OF ITS ABSOLUTE VALUES
}

\author{
DIETER SCHMERSAU \\ Mathematisches Institut der Freien Universität Berlin \\ FB 19, WE 1, Hüttenweg 9 \\ 1000 Berlin 33 \\ (Received March 4, 1981)
}

ABSTRACT. This paper is concerned with a special class of holomorphic functions with extremal properties of its absolute values on arbitrary closed line segments in the complex plane. The main result is a geometrical characterization of the functions $z \rightarrow e^{a z+b}, z \rightarrow(a z+b)^{n}$ and $z \rightarrow(a z+b)^{\alpha+i \beta}$ with $a, b \in \mathbb{c}, \alpha, \beta \in \mathbb{R}, n \in \mathbf{z}$.

KEY WORDS AND PHRASES. Maximum respectively minimum of the absolute value $|f|$ is taken on at one of the endpoints of every closed line segment.

1980 MATHEMATICS SUBJECT CLASSIFICATION CODE. $\quad 30 C 45$

\section{INTRODUCTION.}

The present work is closely related to the following problem raised by Rubel [1]: Find all entire functions $f$ such that for every closed line segment $L$ in the complex plane, wherever located an in whatever direction, the maximum of $|f|$ on $L$ is taken on at one of the two endpoints of $L$.

As a secondary result of the solution for this problem we will obtain a simple characterization of the entire function $z \rightarrow e^{a z+b}$. Suppose $f: G \rightarrow \mathbb{C}$ is a complex function holomorphic in the region $G$. 
Then for all $z=x+i y \in G$ with $f(z) \neq 0$ the partial derivatives of first and second order of the function $w(x, y)=|f(z)|$ are given by [2] :

$$
\begin{aligned}
& w_{x}=|f(z)| \operatorname{Re}\left(\frac{f^{\prime}(z)}{f(z)}\right), w_{y}=-|f(z)| \operatorname{Im}\left(\frac{f^{\prime}(z)}{f(z)}\right\}, \\
& w_{X X}=|f(z)|\left\{\operatorname{Im}^{2}\left(\frac{f^{\prime}(z)}{f(z)}\right)+\operatorname{Re}\left(\frac{f^{\prime \prime}(z)}{f(z)}\right)\right\}, \\
& w_{Y y}=|f(z)|\left\{\operatorname{Re}^{2}\left(\frac{f^{\prime}(z)}{f(z)}\right)-\operatorname{Re}\left(\frac{f^{\prime \prime}(z)}{f(z)}\right)\right\}, \\
& w_{X Y}=|f(z)|\left\{\operatorname{Im}\left(\frac{f^{\prime}(z)}{f(z)}\right) \operatorname{Re}\left(\frac{f^{\prime}(z)}{f(z)}\right)-\operatorname{Im}\left(\frac{f^{\prime \prime}(z)}{f(z)}\right)\right\} .
\end{aligned}
$$

Moreover the formula of Taylor implies:

$$
\begin{aligned}
w(x+h, y+k) & =w(x, y)+h w_{x}(x, y)+k w_{y}(x, y) \\
& +\frac{1}{2}\left\{h^{2} w_{x x}(x, y)+2 h k w_{x y}(x, y)+k^{2} w_{y y}(x, y)\right\} \\
& +o\left(h^{2}+k^{2}\right) .
\end{aligned}
$$

Introducing the variable $\zeta:=h+i k$ we deduce from (1.1) and (1.2):

$$
\begin{aligned}
|f(z+\zeta)|-|f(z)|=|f(z)| & \left\{\operatorname{Re}\left(\frac{f^{\prime}(z)}{f(z)} \zeta\right)+\frac{1}{2} \operatorname{Im}^{2}\left\{\frac{f^{\prime}(z)}{f(z)} \zeta\right)+\frac{1}{2} \operatorname{Re}\left(\frac{f^{\prime \prime}(z)}{f(z)} \zeta^{2}\right\}\right\} \\
& +o\left(|\zeta|^{2}\right) .
\end{aligned}
$$

By means of this equation we prove the following Lemma.

LEMMA 1. Let $\mathrm{f}: \mathrm{G} \rightarrow \mathbb{C}$ be holomorphic in the region $G, z \in G$ with $f(z) \neq 0, f^{\prime}(z) \neq 0$ and

$$
\operatorname{Re}\left(\frac{f(z) f^{\prime \prime}(z)}{f^{\prime 2}(z)}\right)>1 \text { respectively } \operatorname{Re}\left(\frac{f(z) f^{\prime \prime}(z)}{f^{\prime 2}(z)}\right)<1 .
$$

Then there exists a line segment $L$ through $z$ such that $|f|$ does not reach its maximum respectively minimum at one of the endpoints of 
L .

$$
\text { PROOF. Suppose } z \in G \text { with } f(z) \neq 0, f^{\prime}(z) \neq 0
$$

and $\operatorname{Re}\left(\frac{f(z) f^{\prime \prime}(z)}{f^{\prime 2}(z)}\right\}>1$

For real $t$ in a sufficiently small neighbourhood of zero, we define $\zeta:=i \frac{f(z)}{f^{\prime}(z)} t$.

Then we have:

$$
\begin{aligned}
& \operatorname{Re}\left(\frac{f^{\prime}(z)}{f(z)} \zeta\right)=0, \operatorname{Im}\left(\frac{f^{\prime}(z)}{f(z)} \zeta\right)=t, \\
& \operatorname{Re}\left(\frac{f^{\prime \prime}(z)}{f(z)} \zeta^{2}\right)=-t^{2} \operatorname{Re}\left(\frac{f(z) f^{\prime \prime}(z)}{f^{\prime 2}(z)}\right) .
\end{aligned}
$$

From these equations it follows by means of (1.3)

$$
\left|f\left(z+i \frac{f(z)}{f^{\prime}(z)} t\right)\right|-|f(z)|=\frac{t^{2}}{2}|f(z)|\left(1-\operatorname{Re}\left(\frac{f(z) f^{\prime \prime}(z)}{f^{\prime 2}(z)}\right)\right)+o\left(t^{2}\right) \text {. }
$$

Hence there exists a $t_{0} \in R$ such sthat:

$$
\left|f\left(z+i \frac{f(z)}{f^{\prime}(z)} t_{0}\right)\right|<|f(z)| \text { and }
$$$$
\left|f\left(z-i \frac{f(z)}{f^{\prime}(z)} t_{0}\right)\right|<|f(z)|
$$

The case $\operatorname{Re}\left(\frac{f(z) f^{\prime \prime}(z)}{f^{\prime 2}(z)}\right)<1$ is treated in the same way.

The next Lemma is an immediate consequence of the well known theorem of Picard [3], "Let $g$ be a meromorphic function in the whole complex plane. If there exist three different numbers not belonging to the range of $g$, then $g$ is constant".

LEMMA 2. Let $f$ be a meromorphic function in the whole complex plane, which is not constant.

Then the function $g:=\frac{f f^{\prime \prime}}{f^{2}}$ is either a constant or there exist $z_{\circ} z_{1} \in \mathbb{C}$ with 


$$
\operatorname{Re}\left(g\left(z_{0}\right)\right)>1 \text { and } \operatorname{Re}\left(g\left(z_{1}\right)\right)<1 \text {. }
$$

PROOF. Since $f$ is meromorphic in all of $\mathfrak{c}$ and not constant, also the function $g=\frac{f f^{\prime \prime}}{f^{2}}$ is meromorphic in all of $\mathbb{c}$. Then our Lemma immediately follows from the theorem of Picard. Collecting the results obtained so far we end up with the following theorem:

THEOREM 1. Suppose $f$ is a non constant function meromorphic in the whole complex plane such that also $g=\frac{f f^{\prime \prime}}{f^{2}}$ is not a constant. Then there exist two line segments $L_{0}$ and $L_{1}$ such that neither the maximum of $|f|$ on $L_{0}$ nor the minimum of $|f|$ on $L_{1}$ is taken on at the endpoints of these segments.

Next we consider the case that the expression $\frac{f f^{\prime \prime}}{f^{2}}$ is a constant on c .

LEMMA 3. Let $\mathbf{c}=\gamma+i \delta$ be an arbitrary complex number. Then the solutions of the differential equation

$$
\frac{f f^{\prime \prime}}{f \prime^{2}}=c
$$

are giben by:

$$
f(z)=\left\{\begin{array}{l}
e^{a z+b} \text { for } c=1, \\
(a z+b)^{\frac{1}{1-c}} \text { for } c \neq 1 .
\end{array}\right.
$$

PROOF. Rewriting the differential equation (1.5) in the form

$$
\frac{f^{\prime \prime}}{f^{\prime}}=c \frac{f^{\prime}}{f} \text {, }
$$

it may easily be integrated [4].

The result is (1.6) with $a \in \mathbb{C}\{0\}$ and $b \in \mathbb{C}$. In the case $c=\gamma+i \delta \neq 1$ the introduction of new variables $\alpha$ and $\beta$ by $\alpha+i \beta:=\frac{1}{1-c}$ leads to the relations 


$$
\alpha=\frac{1-\gamma}{(1-\gamma)^{2}+\delta^{2}}, \beta=\frac{\delta}{(1-\gamma)^{2}+\delta^{2}}
$$

and thereby

$$
\alpha=0 \leftrightarrow \gamma=1, \alpha>0 \Leftrightarrow \gamma<1, \alpha<0 \Leftrightarrow 1<\gamma,
$$

which will be needed later on.

The investigation of the functions $f(z)=e^{z}$ respectively $f(z)=z$ with regard to the extremal properties of their absolute values causes no difficulties. Since the simple similarity transformation $z \rightarrow a z+b$ maps line segments into line segments there directly follows:

THEOREM 2. If $f$ is a non-constant, entire function on $\mathbb{c}$ such that on every line segment $I$ its absolute value $|f|$ takes on its maximum at one of the endpoints of $L$, then $f$ is given either by $f(z)=e^{a z+b}$ or by $f(z)=(a z+b)^{n}, n \in \mathbb{N}$.

Theorem 2 completely solves the problem of Rubel mentioned at the beginning. In view of the equation $\left|\frac{1}{f(z)}\right|=\frac{1}{|f(z)|}$, a further consequence of Theorem 2 is:

THEOREM 3. If $f$ is a non constant function meromorphic in the entire complex plane such that on every line segment $L$ its absolute value $|f|$ reaches its minimum at one of the endpoints of $L$, then $f$ is given either by $f(z)=e^{a z+b}$ or by $f(z)=\frac{1}{(a z+b)^{n}}, n \in \mathbb{N}$. The combination of theorem 2 and Theorem 3 leads to a simple characterization of the exponential function:

THEOREM 4. Let $f$ be a non-constant entire function such that on every line segment $L$ the absolute value $|f|$ reaches its maximum as well as its minimum at the endpoints of $L$, then $f$ is an exponential function of the form $f(z)=e^{a z+b}$.

In view of Lemma 3 it seems to be interesting to investigate the gen- 
eral power function $f(z)=z^{\alpha+i \beta}, \alpha+i \beta \notin z$, with regard to the extremal properties of its absolute value in the region

$$
G:=\{z \in \mathbb{C} \backslash\{0\} /-\pi<\arg z<\pi\}
$$

Introducing polar coordinates $z=r e^{i \varphi}$, the absolute value of $f$ reads :

$$
|f(z)|=r^{\alpha} \cdot e^{-\beta \varphi}
$$

On the half-lines with $\varphi=$ const. the behaviour of $|f|$ is obvious. In the case of straight lines not running through the origin we have to consider separately those cutting the negative real axis. Finally in view of (1.11) it suffices to investigate $|f|$ on straight lines cutting the positive real axis vertically respectively on half-lines cutting the negative real axis vertically.

A straight line of the first kind is given in polar coordinate by:

$$
r=\frac{p}{\cos \varphi}, p>0,-\frac{\pi}{2}<\varphi<\frac{\pi}{2}
$$

For $\alpha>0$ it follows by means of elementary analysis that there exists exactly one minimum of $|f|$ on (1.12) given by:

$$
\tan \varphi_{1}=\frac{\beta}{\alpha}
$$

Similarly for $\alpha<0$ there exists exactly one maximum of $|f|$ on (1.12) also fixed by (1.13). These results are in accordance with Lemma 1 and equation (1.9). Moreover the result (1.13) may be easily derived also via geometrical arguments by considering the geometry of the set of curves $r^{\alpha} \cdot e^{-\beta \varphi}=$ const. For $1>\alpha, \alpha \neq 0$ there occur two turning points, the position of which is fixed by: 


$$
\tan \varphi_{2,3}=\frac{\beta}{\alpha} \pm \frac{1}{\alpha} \sqrt{\frac{\alpha^{2}+\beta^{2}}{1-\alpha}}
$$

On the half-lines mentioned above the arguments have to be slightly modified because of the limits:

$$
\lim _{\arg \rightarrow \pi}|f(z)|=r^{\alpha} \cdot e^{-\beta \pi}, \underset{\arg z \rightarrow-\pi}{\lim }|f(z)|=r^{\alpha} \cdot e^{\beta \pi} .
$$

Our final result reads:

THEOREM 5. Let $\mathrm{G}$ be the region defined by $(1.10), \mathrm{K}$ the class of all functions $f$ holomorphic and non-constant in $G$ with the further property that $g=\frac{f f^{\prime \prime}}{f^{\prime 2}}$ is meromorphic in the entire complex plane. Every function $f \in K$ such that on any line segment $L \subset G$ its absolute value $|f|$ reaches its maximum (respectively minimum) in one of the endpoints of $L$ is given by

$$
\begin{aligned}
& f(z)=e^{a z+b} \text { or } f(z)=(a z+b)^{\alpha+i \beta} \text { with } \alpha \geq 0 \\
& \text { (respectively } \left.f(z)=e^{a z+b} \text { or } f(z)=(a z+b)^{\alpha+i \beta} \text { with } \alpha \leq 0\right)
\end{aligned}
$$

In passing it should be mentioned that. Ullrich [4] in his paper "Betragflächen mit ausgezeichnetem Krümmungsverhalten" ends up with the same functions which I have discussed in my paper [5], too.

\section{REFERENCES}

1. RUBEL, L.A. Problem 6279, Am. Math. Monthly, Vol. 86, No. 9, 1979.

2. ZAAT, J. Differentialgeometrie der Betragflächen analytischer Funktionen, Mitt. d. Math. Sem. d. Univ. Gießen, 30, 1944.

3. CARATHEODORY, C. Funktionentheorie II, 2. Aufl., Basel, 1961.

4. ULLRICH, E. Betragflächen mit ausgezeichnetem Krümmungsverhalten, Math. Zeitschr., 54, 1951.

5. SCHMERSAU, D. Geometrische Untersuchungen der Betragflächen holomorpher Funktionen, Diss., Berlin, 1977. 


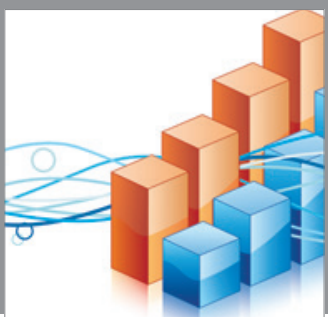

Advances in

Operations Research

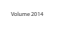

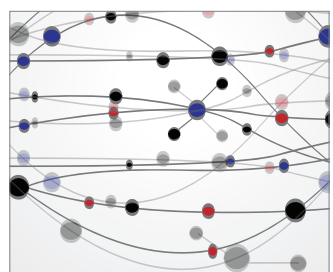

\section{The Scientific} World Journal
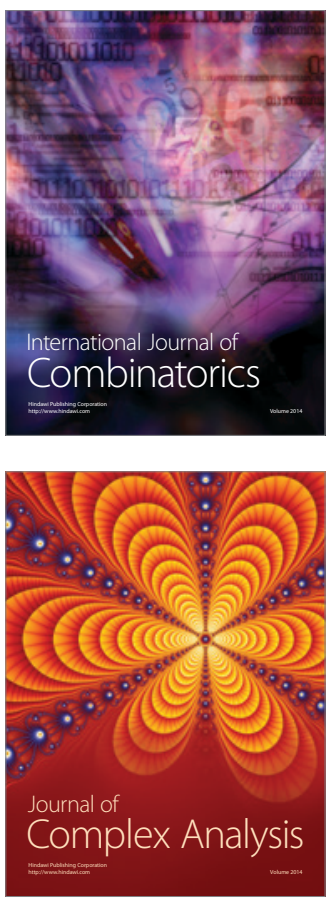

International Journal of

Mathematics and

Mathematical

Sciences
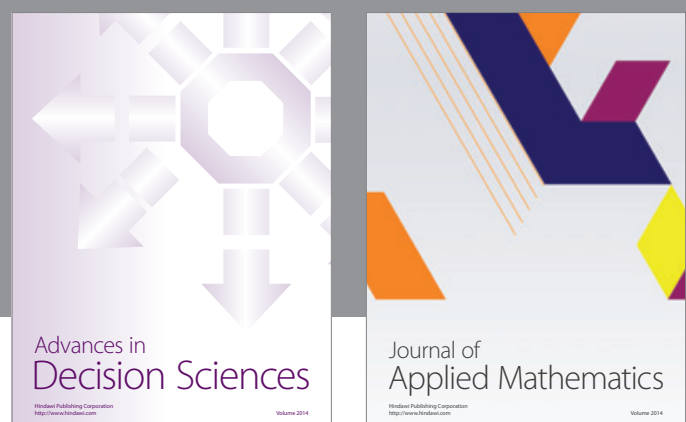

Journal of

Applied Mathematics
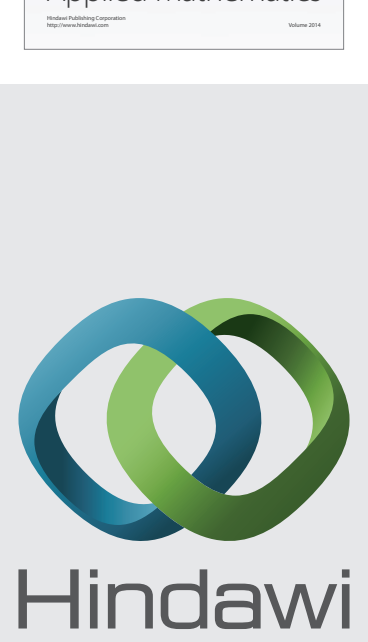

Submit your manuscripts at http://www.hindawi.com
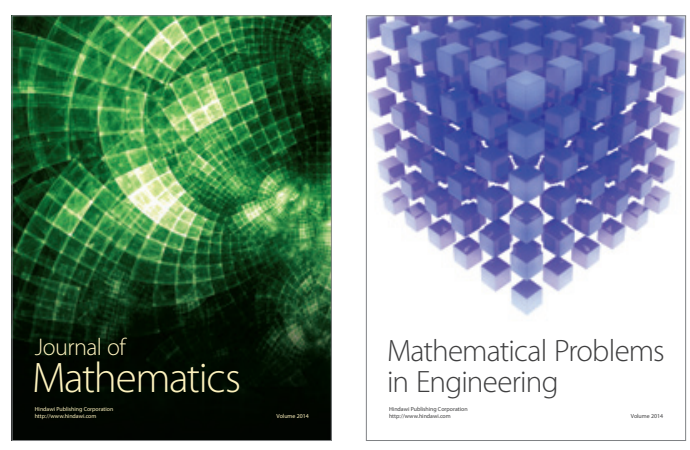

Mathematical Problems in Engineering
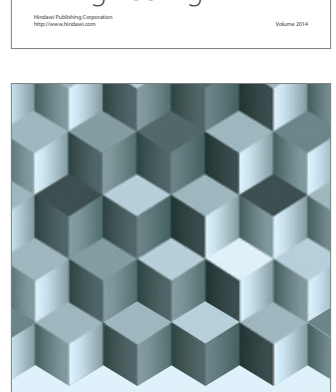

Journal of

Function Spaces
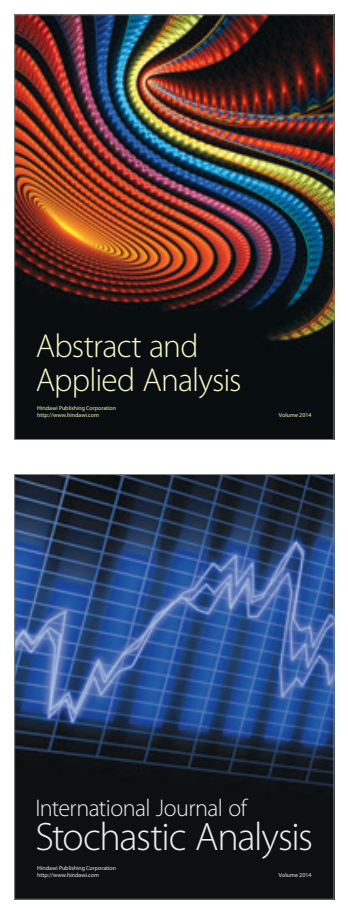

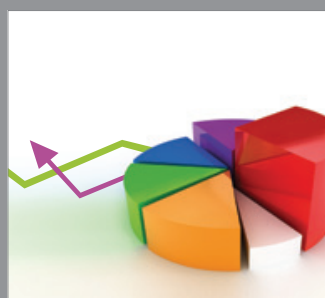

ournal of

Probability and Statistics

Promensencen
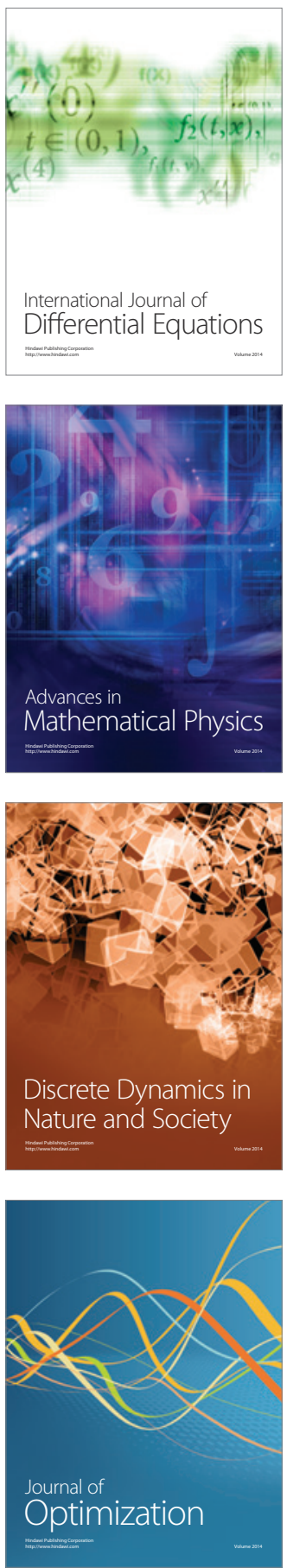